J. Austral-Math. Soc. 19 (Series A) (1975), 129-145.

\title{
A CLASS OF SIMPLE GROUPS WITH NO DOUBLY TRANSITIVE REPRESENTATIONS

\author{
R. J. CLARKE
}

(Received 4 May 1970)

Communicated by G. E. Wall

\section{Introduction}

The first counterexample to the conjecture that all non abelian simple groups have doubly transitive permutation representations was pointed out by Parker in $(1954)$, where he showed that the unitary group $P S U(4,4)$ had no doubly transitive representations. In this paper we generalize Parker's result to give an infinite class of simple groups having no doubly transitive permutation representations. Specifically, we prove

THEOREM 1. The projective symplectic group PSp $(4, q)$ has no doubly transitive permutation representation for $q>2$.

Using the results of Srinivasan (1968) on characters of symplectic groups one could prove this result quite quickly. However, it may be of interest to give a proof not relying on the character table of $S p(4, q)$. Our proof may be generalized to deal with a slightly larger class of groups.

\section{Notation and Preliminaries}

Throughout this paper $G^{*}$ will denote the symplectic group $S p(4, q)$, the subgroup of the general linear group $G L(4, q)$ consisting of all matrices $A$ satisying $A^{\prime} J A=J$, where

$$
J=\left[\begin{array}{rrrr}
0 & 1 & 0 & 0 \\
-1 & 0 & 0 & 0 \\
0 & 0 & 0 & 1 \\
0 & 0 & -1 & 0
\end{array}\right]
$$

We write $q=p^{1}$ with $p$ prime. 
Factoring this group by its centre, the subgroup of scalar matrices, gives the projective symplectic group $G=P S p(4, q)$. We denote elements of $G$ by matrices surmounted by a bar.

The order of $G^{*}$ is $q^{4}\left(q^{2}-1\right)\left(q^{4}-1\right)$ and that of $G$ is $q^{4}\left(q^{2}-1\right)\left(q^{4}-1\right) / d$, where $d=(2, q-1)$.

If $H$ is any finite group, a character of $H$ means a non-negative integral combination of the complex irreducible characters of $H$. The trivial character of $H$ is denoted by $1_{H}$. If $H$ is a subgroup of $G$ and $\phi$ is a character of $H, \phi^{G}$ denotes the character of $G$ induced from $\phi$.

If $S \subset H$, the centralizer of $S$ is denoted by $C_{H}(S)$ and the normalizer of $S$ by $N_{H}(S)$. Finally, the order of any set $X$ is denoted by $|X|$.

We now give various small results which we shall require.

LEMMA 1. Let $A \in K=G L(m, q)$ have the following form:

$$
A=\left[\begin{array}{lllll}
C_{1} & & & \\
& \cdot & & & \\
& & \cdot & & \\
& & & C_{r}
\end{array}\right],
$$

each $C_{i} \in G L\left(m_{i}, q\right), C_{i}$ and $C_{j}$ for $i \neq j$ not conjugate in any linear group and each $C_{i}$ of order $r_{i}$ dividing $q^{m_{i}}-1$ but not dividing

$$
\begin{aligned}
& \prod_{l=1}^{m_{i}-1}\left(q^{l}-1\right) . \quad \text { Then } \\
& \left|C_{K}(A)\right|=\prod_{i}\left(q^{m_{t}}-1\right) .
\end{aligned}
$$

Proof. By considering the possible conjugacy classes of matrices of order prime to $q$ we see that $C_{i}$ is a power of an element of $G L\left(m_{i}, q\right)$ of order $q^{m_{i}}-1$. The result now follows from Schur's lemma and Theorem 7.3 on page 187 of Huppert (1967).

LEMMA 2. Let $K$ be a finite group having a $k$-ply transitive permutation representation of degree $n$ on a set $\Omega$. Let $L$ be the subgroup of $K$ fixing $k$ points and let $U$ be a subgroup of $L$. Let $\Gamma$ be the subset of $\Omega$ consisting of all point fixed by $U$ and suppose that $|\Gamma|=m$. Then

$$
\left|N_{K}(U)\right| \leqq m(m-1) \cdots(m-k+1)\left|N_{L}(U)\right| .
$$

Equality holds if and only if every subgroup of $L$ conjugate to $U$ in $G$ is conjugate to $U$ in L. In this case

$$
\left(K: N_{K}(U)\right)=\left(L: N_{L}(U)\right) \frac{n(n-1) \cdots(n-k+1)}{m(m-1) \cdots(m-k+1)},
$$

and $N_{K}(U)$ acts $k$ - ply transitively on the set $\Gamma$. 
Proof. The first statement results from the fact that $N_{K}(U)$ acts as a group of permutations on $\Gamma$, and $N_{L}(U)$ is the subgroup of $N_{K}(U)$ fixing $k$ points. The second part is the Lemma of Witt (1937; Satz 3.).

Clearly, if $U$ is pronormal in $K$ the conditions of equality hold. So equality holds if $U$ is a Sylow subgroup of $L$.

LemMa 3. Let the finite group $K$ have a doubly transitive permutation representation of degree $n$ on a set $\Omega$. Let $\alpha, \beta \in \Omega$ and write $K_{\alpha \beta}$ for the subgroup of $K$ fixing $\alpha$ and $\beta$. Suppose there exists a prime $p$ dividing $n-1$ and $\left|K_{\alpha \beta}\right|$. If $Q$ is a Sylow p-subgroup of $K_{\alpha \beta}$ then $Q=0_{p}\left(N_{K}(Q)\right)$, that is $Q$ is the maximal normal p-subgroup of its normalizer.

Proof. Let $P=0_{p}\left(N_{K}(Q)\right)$ and suppose $Q$ fixes exactly $m$ points of $\Omega$. $N_{K}(Q)$ acts doubly transitively on these $m$ points and, as $P \triangleleft N_{K}(Q), P$ acts either trivially or transitively on them. Now $m \equiv n \equiv 1(\bmod p)$. Hence $P$, being a $p$ - group, cannot act transitively on $m$ points. Hence $P$ acts trivially on them, which means $P \subset K_{\alpha \beta}$. Thus $P=P \cap K_{\alpha \beta}=Q$.

LEMMA 4. Let a group $K$ have a permutation representation on a set $\Omega$ and let $\Gamma$ be an orbit of some $y \in K$. Let $s$ be a power of a prime $s_{0}$ such that $s$ divides the order of $y$ and let $y^{n}$ have order s. Then if $y^{n}$ fixes any point of $\Gamma$ it fixes all points of $\Gamma$, while otherwise $s_{0} \| \Gamma \mid$.

The proof of this lemma is straightforward.

\section{Symplectic Groups as Chevalley Groups}

$G$ is isomorphic to the Chevalley group $C_{2}(q)$, and we identify the two groups. We shall use freely the papers of Carter (1965) and Tits (1964) on Lie algebras, Chevalley groups and groups with a $B N$-pair. We shall also need the following results of Curtis (1966).

THEOREM A. Let $G$ be the Chevalley group $L(q)$. Let $\Sigma$ be the set of fundamental roots of $L$ and $\$, K \subset \Sigma$. Define the subgroup $W_{J}$ of $W$ to be the group generated by the fundamental reflections for the roots in $J$ and put $G_{J}=B W_{J} B$. Write $\psi_{J}=\left(1_{W J}\right)^{W}$ and $\chi_{J}=\left(1_{G J}\right)^{G}$. Then the mapping

$$
\theta: \psi=\sum_{J} a_{J} \psi_{J} \rightarrow \chi=\sum_{J} a_{J} \chi_{J}
$$

is an isometry between the complex vector spaces generated by the $\psi_{J}$ and the $\chi_{J}$. In fact the scalar product

$$
\begin{aligned}
\left(\chi_{J}, \chi_{K}\right) & =\text { number of }\left(G_{J}, G_{K}\right) \text { double cosets in } G \\
& =\text { number of }\left(W_{J}, W_{K}\right) \text { double cosets in } W \\
& =\left(\psi_{J}, \psi_{K}\right) .
\end{aligned}
$$


The Lie algebra $C_{2}$ has fundamental roots $p_{1}$ and $p_{2}$ and positive roots $p_{1}, p_{2}, p_{1}+p_{2}$ and $2 p_{1}+p_{2}$. For the corresponding elements $x_{r}(t)$ of $C_{2}(q)$ we may write

$$
\begin{aligned}
& x_{p_{1}}(t)=\left[\begin{array}{rrrr}
\overline{1} & 0 & t & 0 \\
0 & 1 & 0 & 0 \\
0 & 0 & 1 & 0 \\
0 & -t & 0 & 1
\end{array}\right], \quad x_{p_{2}}(t)=\left[\begin{array}{llll}
1 & 0 & 0 & 0 \\
0 & 1 & 0 & 0 \\
0 & 0 & 1 & t \\
0 & 0 & 0 & 1
\end{array}\right], \\
& x_{p_{1}+p_{2}}(t)=\left[\begin{array}{rrrr}
1 & 0 & t & 0 \\
0 & 1 & 0 & 0 \\
0 & 0 & 1 & 0 \\
0 & -t & 0 & 1
\end{array}\right], \quad x_{2 p_{1}+p_{2}}(t)=\left[\begin{array}{llll}
1 & t & 0 & 0 \\
0 & 1 & 0 & 0 \\
0 & 0 & 1 & 0 \\
0 & 0 & 0 & 1
\end{array}\right],
\end{aligned}
$$

and

$$
x_{-r}(t)=\left(x_{r}(t)\right)^{\prime}
$$

The subgroup $U$ generated by the root subgroup $X_{r}$, for $r$ a positive root, is a Sylow $p$-subgroup of $G .|U|=q^{4}$. The subgroup $B=N_{G}(U)$ has order $q^{4}(q-1)^{2} / d . B=U H$, where $H$ is the subgroup of $G$ consisting of elements of form

$$
\left[\begin{array}{llll}
\lambda & 0 & 0 & 0 \\
0 & \lambda^{-1} & 0 & 0 \\
0 & 0 & \mu & 0 \\
0 & 0 & 0 & \mu^{-1}
\end{array}\right]
$$

where $\lambda$ and $\mu$ are non zero elements of $G F(q)$.

The Weyl group $W$ of $C_{2}$ is $\left\langle\omega_{1}, \omega_{2} ; \omega_{1}=\omega_{2}^{2}=\left(\omega_{1} \omega_{2}\right)^{4}=1\right\rangle$. Here

$$
\begin{aligned}
& \omega_{1}\left(p_{1}\right)=-p_{1}, \omega_{1}\left(p_{2}\right)=2 p_{1}+p_{2} \\
& \omega_{2}\left(p_{1}\right)=p_{1}+p_{2}, \omega_{2}\left(p_{2}\right)=-p_{2} .
\end{aligned}
$$

We now apply Theorem $A$ to find some characters of $G$.

They Weyl group of $C_{2}$ has subgroups $W_{\phi}=\{1\}, W_{\Sigma}=W, W_{\left\{p_{1}\right\}}=\left\langle\omega_{1}\right\rangle$ and $W_{\left\{p_{2}\right\}}=\left\langle\omega_{2}\right\rangle$.Using the notation of Theorem A, write $\psi_{1}$ for $\psi_{\left\{p_{1}\right\}}$, and so on. The conjugacy classes of $W$ are $C_{0}=\{1\}, C_{1}=\left\{\left(\omega_{1} \omega_{2}\right)^{2}\right\}, C_{2}=\left\{\omega_{1} \omega_{2}, \omega_{2} \omega_{1}\right\}$, $C_{3}=\left\{\omega_{1}, \omega_{2} \omega_{1} \omega_{2}\right\}$ and $C_{4}=\left\{\omega_{2}, \omega_{1} \omega_{2} \omega_{1}\right\}$. For the characters $\psi_{J}$, 


\begin{tabular}{l|ccccc} 
& $C_{0}$ & $C_{1}$ & $C_{2}$ & $C_{3}$ & $C_{4}$ \\
\hline$\psi_{\Sigma}$ & 1 & 1 & 1 & 1 & 1 \\
$\psi_{1}$ & 4 & 0 & 0 & 2 & 0 \\
$\psi_{2}$ & 4 & 0 & 0 & 0 & 2 \\
$\psi_{\phi}$ & 8 & 0 & 0 & 0 & 0
\end{tabular}

The entries in the following table are the scalar products of these characters.

\begin{tabular}{l|cccc} 
& $\psi_{\Sigma}$ & $\psi_{1}$ & $\psi_{1}$ & $\psi_{\phi}$ \\
\hline$\psi_{\Sigma}$ & 1 & 1 & 1 & 1 \\
$\psi_{1}$ & 1 & 3 & 2 & 4 \\
$\psi_{2}$ & 1 & 2 & 3 & 4 \\
$\psi_{\phi}$ & 1 & 4 & 4 & 8
\end{tabular}

By Theorem A a similar table is valid for the characters $\chi_{\Sigma}, \chi_{1}, \chi_{2}$ and $\chi_{\phi}$ of $G$. Using it we see that there are irreducible characters $\phi, \psi, \psi^{\prime}$ and $\chi$ of $G$ such that

and

$$
\begin{aligned}
& \chi_{\Sigma}=1_{G} \\
& \chi_{1}=1_{G}+\phi+\psi \\
& \chi_{2}=1_{G}+\phi+\psi^{\prime}
\end{aligned}
$$$$
\chi_{\phi}=1_{G}+2 \phi+\psi+\psi^{\prime}+\chi \text {, }
$$

so that

$$
\chi=\chi_{\phi}-\chi_{1}-\chi_{2}+\chi_{\Sigma}
$$

It is well known and easy to show that $\operatorname{deg} \chi=q^{4}$.

Consider $K=G_{\left(p_{2}\right)}=\rho B, n_{w} \sigma$. By Carter (1965; page 214) we have

$$
\begin{aligned}
n_{\omega_{2}} & =x_{p_{2}}(1) x_{-p_{2}}(-1) x_{p_{2}}(1) \\
& =\left[\begin{array}{rrrr}
1 & 0 & 0 & 0 \\
0 & 1 & 0 & 0 \\
0 & 0 & 0 & 1 \\
0 & 0 & -1 & 0
\end{array}\right] .
\end{aligned}
$$

Now $G$ may be considered as the group of all collineations of a 3 dimensional projective space $P$ over $G F(q)$ which commute with a certain skew symmetric form on $P$. We see from the form of $B$ and $n_{\omega_{2}}$ that $K$ fixes the point 


$$
x=\left[\begin{array}{l}
1 \\
0 \\
0 \\
0
\end{array}\right]
$$

of $P$. As $K$ is maximal, it must be the subgroup of $G$ fixing $x$. Now $G$ has rank 3 action on $P$. The orbits of $K$ consists of the point $x$, the $q+q^{2}$ points other than $x$ on the orthogonal hyperplane to $x$ and the $q^{3}$ points of $P$ outside this hyperplane. See for these results Higman and McLaughlin (1965).

We shall use the results of Higman (1964) to obtain the degrees of characters $\phi$ and $\psi^{\prime}$, the non trivial irreducible constituents of $\left(1_{K}\right)^{G}$.

In the notation of Higman (1964; page 146), $k=q+q^{2}, l=q^{1}$ and $k<l$.

We calculate the parameters $\lambda$ and $\mu$. We have by Higman (1964; page 148),

$$
\mu l=k(k-\lambda-1) \text {. }
$$

Hence

and

$$
\mu q^{3}=\left(q+q^{2}-\lambda-1\right)
$$

$$
q^{2} \mid(q-\lambda-1) \text {. }
$$

As $\lambda \leqq k, \lambda=q+q^{2}-1$ or $\lambda=q-1$. In the former case $\mu=0$. But as $K$ is maximal in $G$, the rank 3 representation of $G$ is primitive. Hence $\mu \neq 0$ Higman (1964; page 149). So we have

$$
\lambda=q-1, \mu=q+1 .
$$

Write $D=(\lambda-\mu)^{2}+4(k-\mu)=4 q^{2}$. For the degrees $f_{2}$ and $f_{3}$ of $\phi$ and $\psi^{\prime}$ we have in some order

$$
\begin{aligned}
f_{2}, f_{3} & =[2 k+(\lambda-\mu)(k+l) \mp \sqrt{D}(k+l)] /(\mp 2 \sqrt{D}) \\
& = \pm \frac{1}{2} q^{2}+\frac{1}{2} q\left(q^{2}+q+1\right) .
\end{aligned}
$$

Hence as $q>2, p \mid f_{2}, f_{3}$. Thus $p$ divides the degrees of the irreducible non trivial constituents of $\left(1_{B}\right)^{G}$.

\section{The Proof of the Main Theorem}

We prove Theorem 1 in several stages. We have $G=P S p(4, q), q=p^{t}>2$, $p$ a prime, as before. Suppose $G$ has a doubly transitive permutation representation $\rho$ on a set $\Omega$ with $|\Omega|=n$. $G^{*}$ has an action on $\Omega$ via the map $G^{*} \rightarrow G$ which it will at times be convenient to consider. If $\alpha \in \Omega$ and $g \in G$ we write $g \alpha$ for $\rho(g) \alpha$. Let $G_{\alpha}$ be the subgroup of $G$ fixing $\alpha$.

(A) If $p \nmid n$ then $G_{\alpha}$ is a maximal parabolic subgroup of $G$. 
Proor. We recall that a parabolic subgroup of a group $G$ with a $B N$-pair $(B, N)$ is a subgroup conjugate to one containing $B$. As $p \nmid n$ we may take the Sylow $p$-subgroup $U$ of $G$ to be contained in $G_{\alpha}$. As $H G_{\alpha} \geqq H U=B, H G_{\alpha}$ is a parabolic subgroup of $G$. Now $G_{\alpha}$ is maximal in $G$ as $G$ acts doubly transitively on $\Omega$. Hence either $H G_{\alpha}=G_{\alpha}$ or $H G_{\alpha}=G$. In the first case we have the required result. In the second case, $H$ acts transitively on $\Omega$. Now $H$ normalises each root subgroup $X_{r}$, and if $r$ is a positive root, $X_{r} \subset U \subset G_{\alpha}$.

Let $\beta \in \Omega$. Then there is an $h \in H$ such that $\beta=h \alpha$. Then

$$
X_{r}=h X_{r} h^{-1} \subset h G_{\alpha} h^{-1}=G_{\beta}
$$

Thus $X_{\mathrm{r}} \subset \cap_{\beta \in \Omega} G_{b}=\{1\}$, as $G$ is a simple group, contradiction. We note that this result holds for an arbitrary Chevalley group.

(B) $p \mid n$

Proof. If $p \nmid n, G_{\alpha}$ is a maximal parabolic subgroup of $G$. Hence $G_{\alpha}$ is conjugate either to $G_{\left\{p_{1}\right\}}$ or $G_{\left\{p_{2}\right\}}$. But as $G$ acts doubly transitively on $\Omega$, there are two $G_{\alpha}$ double cosets in $G$, that is $\left(1_{G \alpha}\right)^{G}=1_{G}+\zeta$ for some irreducible character $\zeta$. This contradicts our information about the characters $\chi_{1}$ and $\chi_{2}$ above.

(C) $n|| B \mid=q^{4}(q-1)^{2} / d$.

Proof. We have that $\left(1_{G \alpha}\right)^{G}=1_{G}+\zeta$ and

$$
\left(1_{B}\right)^{G}=1_{G}+2 \phi+\psi+\psi^{\prime}+\chi,
$$

where $\zeta, \phi, \psi, \psi^{\prime}$ and $\chi$ are irreducible characters. Now $\zeta$ has degree $n-1$ coprime to $p$. But we know that $\phi, \psi, \psi^{\prime}$ and $\chi$ each have degree divisible by $p$. Hence $\zeta$ is distinct from these characters, so we have

$$
\left(\left(1_{B}\right)^{G},\left(1_{G \alpha}\right)^{G}\right)=1 .
$$

This is equivalent to $B G_{\alpha}=G$. Hence $n=\left(G: G_{\alpha}\right)|| B \mid$.

(D) $n \equiv 1$ or $2\left(\bmod \left(q^{2}+1\right) / d\right)$.

Proof. Write $G L=G L(4, q), \overline{G L}=G L\left(4, q^{4}\right)$ and $\bar{G}=S p\left(4, q^{4}\right)$. Let $\kappa$ be a primitive $q^{4}-1$ th root of unity in the Galois field $G F\left(q^{4}\right)$. Put $\zeta=\kappa^{q 2-1}$. Write

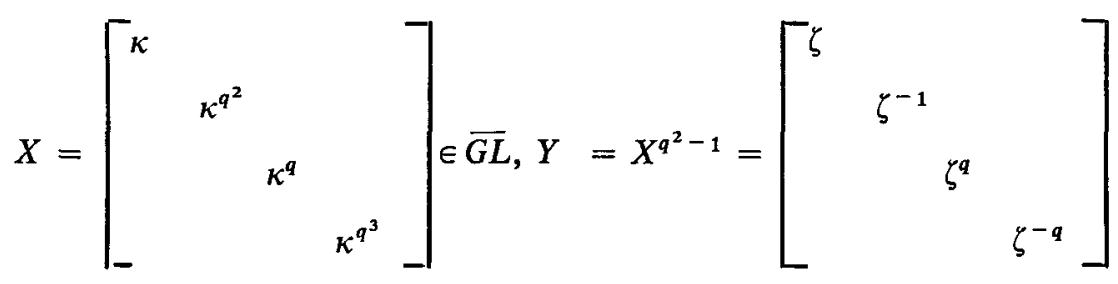


Finally, put

$$
A=\left[\begin{array}{rrrr}
0 & 0 & 1 & 0 \\
0 & 0 & 0 & 1 \\
0 & 1 & 0 & 0 \\
-1 & 0 & 0 & 0
\end{array}\right] \in \bar{G}
$$

We have $A X A^{-1}=X^{q}$. Given any matrix $E \in \overline{G L}$, write $Z^{(q)}$ for the matrix obtained from $Z$ by raising all its entries to the $q$ th power. Using a theorem of Lang (1956) we see that $\exists B \in G$ such that $A^{-1}=B^{-1} B^{(q)}$.

Put $x=B X B^{-1}, y=B Y B^{-1}, a=B A B^{-1}$. Then

$$
\begin{aligned}
x^{(q)} & =B^{(q)} X^{(q)} B^{2(q)} \\
& =B^{(q)} A X A^{-1} B^{-(q)}, \text { as } X^{(q)}=X^{q} \\
& =B X B^{-1} \\
& =x .
\end{aligned}
$$

Thus $x \in G L$. Similarly $y$ and $a \in G L$. In fact $y$ and $a$ are in $G L \cap \bar{G}=G^{*}$.

Write $N=\langle y, a\rangle$. Then $N$ has defining relations

$$
y^{q^{2}+1}=1, \text { aya } a^{-1}=y^{q}, a^{4}=y^{\left(q^{2}+1\right) / d} .
$$

If $b$ is any integer not divisible by $\left(q^{2}+1\right) / d$,

$$
\begin{aligned}
C_{G^{*}}\left(y^{b}\right) & =G^{*} \cap C_{G L}\left(y^{b}\right) \\
& =G^{*} \cap\langle x\rangle, \text { by Lemma } 1, \\
& =\langle y\rangle
\end{aligned}
$$

for $x^{m} \in G^{*}$ if and only if $x^{m} \in \bar{G}$ which happens if and only if $m$ is divisible by $q^{2}-1$, that is $x^{m} \in\langle y\rangle$. Hence $N=N_{G^{*}}\left\langle y^{b}\right\rangle$.

To complete the proof of (D) we consider the action of $G^{*}$ on $\Omega$. Let $s$ be a prime power dividing $\left(q^{2}+1\right) / d$ and write $s=s_{0}^{e}$ for $s_{0}$ a prime. Now $s_{0}$ is prime to $\left(G^{*}:\langle y\rangle\right)$, so the Sylow $s_{0^{-}}$-subgroup of $\langle y\rangle$, which is cyclic, is a Sylow $s_{0^{-}}$ subgroup of $G^{*}$. If $S=\left\langle y^{b}\right\rangle$ is the unique subgroup of order $s$ of $\langle y\rangle, S$ is the unique subgroup of order $s$ of any Sylow subgroup of $G^{*}$ containing $S$. Hence $S$ is a pronormal subgroup of $G^{*}$.

Let $\Gamma_{s}$ be the set of points of $\Omega$ fixed by $S$. If $\left|\Gamma_{s}\right|=m_{s}$ and $m_{s} \geqq 2$, we see from Lemma 2 that $N=N_{G^{*}}(S)$ acts doubly transitively on $\Gamma_{s}$.

LEMMA 5. Let $N$ have a doubly transitive permutation representation $\sigma$ of degree $m$ on a set $\Gamma$ such $\Gamma$ such that $a^{4} \in k e r \sigma$. Then either, 
(i) $m=2$ and $k e r \sigma=\left\langle y, a^{2}\right\rangle$ or

(ii) $m=3$ or $5, m \mid\left(q^{2}+1\right) / d, y^{m} \in$ ker $\sigma$ and $\langle y\rangle$ acts transitively on $\Gamma$.

ProOf. $\sigma(\langle y\rangle)$ is a normal subgroup of a doubly transitive group, so is either trivial or transitive.

If $\sigma(y)=1$, the abelian group $N /\langle y\rangle$ acts doubly transitively on $\Gamma$. Hence $m=2$ and we have case (i).

Suppose $\sigma(\langle y\rangle)$ is transitive. As $\langle y\rangle$ is abelian, $y$ acts regularly on $\Gamma$. Hence $m \mid\left(q^{2}+1\right) / d$ and $y^{m} \in \operatorname{ker} \sigma$. Now $|\sigma(N)|$ divides $\left(N:\left\langle y^{m}, a^{4}\right\rangle\right)=4 m$ and $m(m-1)$ divides $|\sigma(N)|$. Thus $(m-1) \mid 4$ and $m \neq 2$ as $\left(q^{2}+1\right) / d$ is odd. This gives case (ii) and proves the lemma.

We see from Lemma 5 that we have one of the following cases for $m_{s}$ :

(1) $m_{s}=0$.

(2) $m_{s}=1$. Then $y$ fixes exactly one point of $\Omega$.

(3) $m_{s}=2$ and $y$ fixes $\Gamma_{s}$. Then $y$ fixes no other point of $\Gamma$.

(4) $m_{s}=3$ or 5 and $\langle y\rangle$ acts transitively on $\Gamma_{s}$. Then $y$ fixes no points of $\Omega$.

If (1) holds we have from Lemma 4 that $s_{0} \mid n$. But $|B|$ and $\left(q^{2}+1\right) / d$ are coprime, $s_{0} \mid\left(q^{2}+1\right) / d$ and $n|| B \mid$, contradiction.

If (2) holds it is clear that $m_{u}=1$ for every prime power $u$ dividing $\left(q^{2}+1\right) / d$. Let $\Delta$ be an $S$-orbit of $\Omega$ of length greater than one. Suppose $s \nmid|\Delta|$. Then $|\Delta|=s_{0}^{f}$ for some $f<e$. But then $y^{b s_{0} f}$ has order $s_{0}^{e-f}$ and fixes all points of $\Delta$, contradiction. Hence $s \| \Delta \mid$. Thus $s \mid(n-1)$. As this holds for every prime power dividing $\left(q^{2}+1\right) / d$ we have, as required,

$$
\left(q^{2}+1\right) / d \mid(n-1) \text {. }
$$

If (3) holds it is clear that $m_{u}=2$ for every prime power $u$ dividing $\left(q^{2}+1\right) / d$.) Reasoning similar to that of case (2) gives

$$
\left(q^{2}+1\right) / d \mid(n-2) \text {. }
$$

Suppose (4) holds and write $u=m_{s}$. As $u$ is a prime dividing $\left(q^{2}+1\right) / d$, $m_{u}=3$ or 5 . Now $\Gamma_{s}$ and $\Gamma_{u}$ are each $y$-orbits of $\Omega$. We have two cases.

(i) $\Gamma_{s}=\Gamma_{u}$. Then $u=m_{u}$ and, as $u \mid\left(n-m_{u}\right)$ by Lemma 4 , we have $u \mid n$, contradiction.

(ii) $\Gamma_{s} \neq \Gamma_{u}$. Let be the l.c.m. of $m_{s}$ and $m_{u}$. Then $y^{h}$ fixes all points of $\Gamma_{s} \cup \Gamma_{u}$. So no odd prime divides the order of $y^{h}$, for otherwise $y^{h}$ would fix only the points in one $y$-orbit. Thus $y^{2 h}=1$. But then $\left(q^{2}+1\right) \mid 2 h \leqq 2.3 .5$. Hence $q=2$ or 3 . But in neither of these cases is $\left(q^{2}+1\right) / d$ divisible by two distinct primes, contradiction. This completes the proof of $(D)$.

(E) We have the following two possibilities:

(a) $q=3,4,5,8$ or 11 and $n=|\Omega|=\frac{1}{2} q(q-1)^{2}$

(b) $n=|\Omega|=q^{4}$. 
Proof. We have

$$
n \equiv c\left(\bmod \left(q^{2}+1\right) / d\right),
$$

where $c=1$ or $2, q=p^{t}, p$ a prime. We may write

$$
n=q^{i} p^{-b} m
$$

where $1 \leqq i \leqq 4,0 \leqq b<t, p \nmid m$, and

$$
i=2 l-j,
$$

where $1 \leqq l \leqq 2$ and $0 \leqq j<2$. Now

$$
1 / q^{2} \equiv-1\left(\bmod \left(q^{2}+1\right) / d\right) .
$$

Hence

$$
m \equiv n q^{-2 i} q^{j} p^{b} \equiv c(-1)^{l} q^{j} p^{b}\left(\bmod \left(q^{2}+1\right) / d\right) .
$$

Write

$$
m=k\left(q^{2}+1\right) / d+(-1)^{l} c q^{j} p^{b} .
$$

Then $m \mid(q-1)^{2} / d$ from part $(C)$. We must consider various cases.

Case (1): $l=2$. Then $k \leqq 0$.

(i) Suppose $k=0$. Thus

We have $m=c q^{j} p^{b}$ and $m$ is coprime to $p$. Hence $b=j=0$ and $m=c$.

$$
n=c q^{4} \text {. }
$$

If $c=1$ we have (b). If $c=2$,

$$
n-1=\left({ }_{2} q^{4}-1\right) \mid(q-1)^{2}(q+1)^{2}\left(q^{2}+1\right) / d,
$$

since $n(n-1) \| G \mid$. But

$$
n-2=2\left(q^{4}-1\right)=2(q-1)(q+1)\left(q^{2}+1\right) .
$$

Thus $(n-1) \mid 1 / d$. So $n=2$, and $G$ is the cyclic group of order 2 , contradiction.

(ii) Suppose $k<0$.

As $m>0$ we have $q^{2}+1<c d q^{j} p^{b} \leqq c d q^{j+1} / p$. Thus $p<c d q^{j-1}$. The only possibility, as $j \leqq 1$, is $p=3, j=1, c=d=2$ and $b=t-1$. Then

$$
2 m=-\left(q^{2}+1\right)+4 q^{2} / 3=\left(q^{2}-3\right) / 3,
$$

which must divide $(q-1)^{2}$. Therefore $q=3, m=1$ and

$$
n=q^{2 l-j} p^{-b} m=27 .
$$


However then $n-1=26 \times|P S p(4,3)|$, contradiction.

Case (2): $l=1$. Then as $m>0$ we have $k>0$. Now

$$
d m=\left[k\left(q^{2}+1\right)-c d q^{j} p^{b}\right] \mid(q-1)^{2}=q^{2}-2 q+1 .
$$

Hence, as $k \geqq 1,2 q \leqq c d q^{j} p^{b} \leqq c d q^{j+1} / p$. Hence $p \leqq c d q^{j} / 2$, so $j=1$.

(i) Let $k=1$.

$$
d m=\left(q^{2}+1-c d q p^{b}\right) \mid(q-1)^{2} .
$$

Thus $d m \mid(q-1)^{2}-d m=\left(c d p^{b}-2\right) q$. Evidently $c d p^{b} \geqq 2$, for otherwise $d m>(q-1)^{2}$.

( $\alpha$ ) Suppose $c d p^{b}=2$. Then

$$
\begin{aligned}
d m & =q^{2}+1-2 q=(q-1)^{2}, \\
n & =q(q-1)^{2} / d p^{b} .
\end{aligned}
$$

Now if $d p^{b}=1, n=q(q-1)^{2}$. But $(n-1) \| G \mid$, and $n-1$ is coprime to $q(q-1)$ and $q^{2}+1$. Thus $(n-1) \mid(q+1)^{2}$, which is false for all $q>2$. Hence $d p^{b}=2$ and $c=1$. Then $n=\frac{1}{2} q(q-1)^{2}$. As $n(n-1) \| G|,(n-1)|(q+1)^{2}\left(q^{2}+1\right)$. But $n-1=\frac{1}{2}(q-2)\left(q^{2}+1\right)$. Thus $(q-2) \mid 2(q+1)^{2}$. So, as h.c.f. $(q-2, q+1) \mid 3$, $(q-2) \mid 2.3^{2}$. Hence

$$
q=3,4,5,8 \text { or } 11 \text {. }
$$

This gives case (a).

( $\beta$ ) Suppose $c d p^{b}>2$. Then

$$
d m=\left(q^{2}+1-c d p^{b} q\right) \mid\left(c d p^{b}-2\right) q .
$$

But $(d m, q)=1$, so that $d m \mid\left(c d p^{b}-2\right)$. Hence $d m \leqq c d p^{b}-2$. Thus

$$
\left(q^{2}+3\right) /(q+1) \leqq c d p^{b} \leqq c d q / p .
$$

But we have $d m \geqq 0$, so $c d p^{b} \leqq\left(q^{2}+1\right) / q$. Hence

$$
q-1+4 /(q+1) \leqq c d p^{b} \leqq q+1 / q .
$$

Since $c d p^{b}$ is an integer we have $c d p^{b}=q$. Then

$$
d m=q^{2}+1-c d p^{b} q=1 .
$$

Thus $d=m=1$ and $n=m q p^{-b}=c=2$, contradiction.

(ii) Let $k>1$. Then

$$
d m=(k-1)\left(q^{2}+1\right)+q\left(q-c d p^{b}\right)+1 \leqq(q-1)^{2} .
$$


Therefore $q<c d p^{b}$, from which follows $c=d=2, p=3, p^{b}=q / 3$. Then we have

$$
\begin{aligned}
2 m & =2\left(q^{2}+1\right)-4 q^{2} / 3 \\
& =2\left(q^{2}+3\right) / 3 \mid(q-1)^{2} .
\end{aligned}
$$

Now $\left(q^{2}+3, q-1\right) 4$ and so $2\left(q^{2}+3\right) / 3 \mid 4^{2}$, that is $\left(q^{2}+3\right) \mid 24$. But then

$$
2 m=2\left(3^{2}+3\right) / 3 \mid(3-1)^{2}, \text { which is false. }
$$

We have now proven (E). We complete the proof of Theorem 1 by eliminating the remaining possibilities.

1. $\mathbf{q}=3, \mathbf{n}=6$.

$|P S p(4,3)|=263^{4} 5$, and $\left|S_{6}\right|=6 !=2^{4} 3^{2} 5$. If $G$ had a doubly transitive permutation representation of degree 6 it would be isomorphic to a subgroup of $S_{6}$, which it is not.

2. $q=5, n=40$, or $q=11, n=550$.

Let $\alpha$ and $\beta$ be distinct elements of $\Omega$. Then

$$
\begin{aligned}
|G| & =2^{6} 3^{2} 5^{4} 13, q=5, \\
|G| & =2^{6} 3^{2} 5^{2} 11^{4} 61, q=11, \\
\left|G_{\alpha \beta}\right| & =2^{3} 3 \cdot 5^{3}, q=5, \\
\left|G_{\alpha \beta}\right| & =2^{5} 11^{3}, q=11 .
\end{aligned}
$$

Let $Q$ be a Sylow $q$-subgroup of $G_{\alpha \beta}$ and let $Q$ fix exactly $m$ points of $\Omega$. $\left(G_{\alpha}: N_{G_{\alpha}}(Q)\right)=\left(G_{\alpha \beta}: N_{G_{\alpha \beta}}(Q)\right)(n-1) /(m-1)$ is integral and by Sylow's Theorem

$$
\begin{aligned}
& \left(G_{\alpha \beta}: N_{G_{\alpha \beta}}(Q)\right)=1 \text { or } 6, q=5, \\
& \left(G_{\alpha \beta}: N_{G_{\alpha \beta}}(Q)\right)=1, q=11 .
\end{aligned}
$$

Consider $q=11 . m=11 k$ for some $k<50$, and by integrality $(11 k-1)$ $(550-1)$. There is no such $k$. Hence this case does not occur.

Consider $q=5$. $m=5 k$ for some $k<8$, and either $(5 k-1) \mid(40-1)$ or $(5 k-1) \mid 6(40-1)$. 
Hence $k=2$ and $\left(G_{\alpha \beta}: N_{G_{\gamma \beta}}(Q)\right)=6$. Now

$$
\begin{aligned}
\left|N_{G}(Q)\right| & =\left|N_{G_{x \beta}}(Q)\right| m(m-1) \\
& =2^{2} 5^{3} 10 \cdot 9 \\
& =2^{3} 3^{2} 5^{4},
\end{aligned}
$$

by Lemma 2. Now $|Q|=5^{3}$ and $|U|=5^{4}$. Hence we may assume that $Q$ is a normal subgroup of $U$ with cyclic factor group. We have that the derived group $U^{\prime} \subset Q$.

From the Chevalley commutator formula (Carter (1965; page 211)), we have that

So

$$
U^{\prime}=X_{p_{1}+p_{2}} X_{2 p_{1}+p_{2}}
$$

$$
Q=\left\langle U^{\prime}, x_{p_{1}}\left(t_{1}\right) x_{p_{2}}\left(t_{2}\right)\right\rangle,
$$

where $t_{1}, t_{2} \in G F(q)$ are not both zero.

We consider two cases.

(1) Suppose $t_{1} \neq 0$. Then $Q^{\prime}=X_{2 p_{1}+p_{2}}$ and $C_{Q}\left(Q^{\prime}\right)=X_{p_{1}+p_{2}} X_{2_{p_{1}}+p_{2}}$. These groups are each characteristic in $Q$, so

$$
N_{G}(Q) \subset N_{G}\left(X_{2 p_{1}+p_{2}}\right) \cap N_{G}\left(X_{p_{1}+p_{2}} X_{2 p_{1}+p_{2}}\right)=M .
$$

Now using the formula $n_{\omega} X_{r} n_{\omega}^{-1}=X_{\omega(r)}$ of Carter (1965; page 214) we see that $M=B$. Thus

$$
\left|N_{G}(Q) \|\right| B \mid=2^{3} 5^{4}, \text { a contradiction. }
$$

(2) Suppose $t_{1}=0$. Then $Q=X_{p_{1}} X_{p_{1}+p_{2}} X_{2 p_{1}+p_{2}}$. It is easily calculated that

$$
N_{G}(Q)=B \cup B n_{\omega_{1}} B .
$$

Now $\left|B n_{\omega_{1}} B\right|=|B| q^{m}$, where $m$ is the number of positive roots of $C_{2}$ transformed by $\omega_{1}$ into negative roots (Carter (1965; page 220)). Thus

a contradiction.

$$
\begin{aligned}
\left|N_{G}(Q)\right| & =|B|(1+q) \\
& =\frac{1}{2} 5^{4}(5-1)(1+5) \\
& =2^{8} 3 \cdot 5^{4},
\end{aligned}
$$

3. $\mathrm{q}=4, \mathrm{n}=18$

Let $\alpha$ and $\beta$ be distinct elements of $\Omega$. Then

$$
|G|=2^{7} 3^{2} 5^{2} 17,\left|G_{\alpha \beta}\right|=2^{7} 5^{2} .
$$


Let $P$ be a Sylow 5-subgroup of $G_{\alpha \beta}$ and suppose $P$ fixes exactly $m$ points of $\Omega$. Then as $m \equiv 18(\bmod 5), m=3$ or 8 . By Sylow's Theorem $\left(G_{\alpha \beta}: N_{G_{\alpha \beta}}(P)\right)=1$ or $2^{4}$. Now $\left(G_{\alpha}: N_{G_{\alpha}}(P)\right)=\left(G_{\alpha \beta}: N_{G_{\alpha \beta}}(P)\right)(n-1) /(m-1)$ is integral, so we have $m=3$ and $\left.G_{\alpha \beta}: N_{G_{\alpha \beta}}(P)\right)=2^{4}$. By Lemma 2

$$
\begin{aligned}
\left|N_{G}(P)\right| & =\left|N_{G \alpha \beta}(P)\right| m(m-1) \\
& =2^{4} 3 \cdot 5^{2} .
\end{aligned}
$$

Since $G=G^{*}=S p(4,4)$ we are in fact working with matrices. $P$, being a Sylow 5-subgroup of $G$ may be considered as generated by matrices

$$
a=\left[\begin{array}{ll}
A & O \\
O & I_{2}
\end{array}\right], \quad b=\left[\begin{array}{ll}
I_{2} & O \\
O & A
\end{array}\right],
$$

where $A \in S L(2,4)$ has order 5 . By Lemma 1 we have $\left|C_{G L(4,4)}\left(a b^{2}\right)\right|=\left(4^{2}-1\right)^{2}$. In fact the centralizer of $a b^{2}$ consists of matrices

$$
c=\left[\begin{array}{ll}
C & 0 \\
O & D
\end{array}\right]
$$

where $C, D \in C_{G L(2,4)}(A)$, a group generated by a matrix of order 15 with determinant a primitive cube root of unity. Now $c \in G$ if and only if $C, D \in S L(2,4)$. Thus $\left|C_{G}\left(a b^{2}\right)\right|=5^{2}$ and $\left|C_{G}(P)\right|=5^{2}$. So $C_{G}(P)=P$.

The only elements of $P$ with the same eigenvalues as $a$ are $a, b, a^{-1}$ and $b^{-1}$. Thus if $g \in N_{G}(P), g a g^{-1}=a, b, a^{-1}$ or $b^{-1}$. There are the same choices for $g b g^{-1}$. Since $g a g^{-1}$ and $g b g^{-1}$ generate $P$ we see that $\left(N_{G}(P): P\right) \leqq 8$. This is a contradiction.

4. $q=8, n=196$

Let $\alpha$ and $\beta$ be distinct elements of $\Omega$. Then

$$
|G|=2^{12} 3^{4} 5 \cdot 7^{2} 13,\left|G_{\alpha \beta}\right|=2^{10} 3^{3} \text {. }
$$

Let $Q$ be a Sylow 3-subgroup of $G_{\alpha \beta}$ and $P$ a Sylow 3-sub-group of $G$ containing $Q$. We may take $P=\langle a, b\rangle$, where

$$
a=\left[\begin{array}{cc}
A & O \\
O & I_{2}
\end{array}\right], \quad b=\left[\begin{array}{cc}
I_{2} & O \\
O & A
\end{array}\right],
$$

with $A \in S L(2,8)$ an element of order 9 . As in the preceding case we have $C_{G}(P)=P$. In fact $C_{G}\left(a^{i} b^{j}\right)=P$ unless $3|i, 3| j$ or $3 \mid(i-j)$. Clearly $Q$ must contain elements other than those of form $a^{i}, b^{i}$ and $(a b)^{i}$. Hence $C_{G}(Q)=P$. Thus $N_{G}(Q) \subset N_{G}\left(C_{G}(Q)\right)=N_{G}(P)$ and so $P \triangleleft N_{G}(Q)$, contradicting Lemma 3 . 
5. $\mathbf{n}=1$, any $\mathbf{q}$.

Let $\alpha \in \Omega$ and let $U$ be the upper unitriangular subgroup of $G$. $U$ is a Sylow $p$-subgroup of $G$, and $G=U G_{\alpha}$. We take the elements of $U$ as a transversal for $G_{\alpha}$ in $G$.

Let $\theta \in G F(q)$ be of maximal multiplicative order such that

$$
h=\left[\begin{array}{llll}
1 & 0 & 0 & 0 \\
0 & 1 & 0 & 0 \\
0 & 0 & \theta & 0 \\
0 & 0 & 0 & \theta^{-1}
\end{array}\right]
$$

is in some $G_{\alpha} \cdot \theta \neq 1$, as $(q-1)^{2} / d \| G_{\alpha} \mid$. Now $h$ normalizes $U$, so if $u \in U$, $h U G_{\alpha}=h u h^{-1} G_{\alpha}$. Thus the number of points of $\Omega$ fixed by $h$ is $\left|C_{m}(h)\right|$. Put

$$
u=x_{p_{1}}\left(t_{1}\right) x_{p_{2}}\left(t_{2}\right) x_{p_{1}+p_{2}}\left(t_{3}\right) x_{2 p_{1}+p_{2}}\left(t_{4}\right)
$$

Then

$$
h u h^{-1}=x_{p_{1}}\left(\theta^{-1} t_{1}\right) x_{p_{2}}\left(\theta^{2} t_{2}\right) x_{p_{1}+p_{2}}\left(\theta^{-3}\right) x_{2 p_{1}+p_{2}}\left(t_{4}\right)
$$

Thus either $\theta^{2} \neq 1$ and $h$ fixes exactly $q$ points of $\Omega$ or $\theta^{2}=1$ and $h$ fixes exactly $q^{2}$ points of $\Omega$. In the latter case it is clear that $q=3$. We consider this case later.

(1) Suppose $\theta^{2} \neq 1$. Write $S=\langle h\rangle$. By Lemma 2,

$$
\begin{aligned}
\left|N_{G}(S)\right| & \leqq\left|N_{L}(S)\right| q(q-1), L=G_{\alpha \beta}, \beta \neq \alpha \\
& \leqq|L| q(q-1)=q(q-1)^{2}(q+1)^{2} / d
\end{aligned}
$$

But $h$ is centralised by the elements

$$
\left[\begin{array}{lllll}
\hline & & 0 & 0 \\
& & & 0 & 0 \\
0 & 0 & \lambda & 0 \\
0 & 0 & 0 & \lambda^{-1} &
\end{array}\right]
$$

of $G$, where $A \in S L(2, q), \lambda \in G F(q), \lambda \neq 0$. Thus

$$
\begin{aligned}
\left|C_{G}(S)\right| & \geqq(q-1)|\operatorname{PSL}(2, q)| \\
& =q(q-1)^{2}(q+1) / d .
\end{aligned}
$$


Also the element

$$
\left[\begin{array}{rrrr}
1 & 0 & 0 & 0 \\
0 & 1 & 0 & 0 \\
0 & 0 & 0 & 1 \\
0 & 0 & -1 & 0
\end{array}\right]
$$

normalises $S$. Thus we have a contradiction.

(2) Suppose $\theta^{2}=1, q=3$. Then Then $h$ fixes 9 points of $\Omega$ and, writing $S=\langle h\rangle$ and $L=G_{\alpha \beta}$ with $\beta \neq \alpha$, we have as in (1)

$$
\begin{gathered}
\left|N_{G}(S)\right| \leqq|L| 9(9-1) \\
=253^{2} .
\end{gathered}
$$

But $h$ is centralised by the elements

$$
\left[\begin{array}{ll}
\bar{A} & 0 \\
0 & B
\end{array}\right]
$$

of $G$, where $A, B \in S L(2,3)$. Hence

$$
\begin{aligned}
\left|C_{G}(S)\right| & \geqq \frac{1}{2}|S L(2,3)|^{2} \\
& =253^{5} .
\end{aligned}
$$

As in (1) we deduce $\left|N_{G}(S)\right|>23^{5}$, a contradiction. This proves Theorem 1.

Our proof may be generalised to give a similar result for a slightly larger class of groups. In fact one can prove

THEOREM 2. The group $\operatorname{PSp}\left(2^{r}, q\right)$ has no doubly transitive permutation representation for $r \geqq 2$, excepting for each $r$ at most a finite number of values of $q$.

Many parts of the above proof need only slight modification. To prove the corresponding part $(C)$ we need the unpublished result of D. G. Higman that the degrees of the non trivial irreducible constituents of $\left(1_{\beta}\right)^{G}$ are almost always divisible by $p$. More complex manipulation with special subgroups of $G$ is needed to eliminate certain special cases which arise.

The material in this paper was part of my thesis submitted for the degree of $\mathrm{Ph} . \mathrm{D}$. at the University of Warwick. I should like to express my gratitude to Professor J. A. Green for his help and encouragement.

I gratefully acknowledge the support of a British Council Commonwealth Scholarship. 


\section{References}

R. W. Carter (1965), 'Simple groups and simple Lie algebras', J. London Math. Soc. 40, 193-240.

C. W. Curtis (1966), 'The Steinberg character of a finite group with a BN-pair', J. Algebra 4, 433-441.

D. G. Higman (1964), 'Finite permutation groups of rank 3', Math. Zeitschr. 86, 145-156.

D. G. Higman and J. E. McLaughlin (1965), 'Rank 3 subgroups of finite symplectic and unitary groups', $J$, reine u. angew. Math. 218, 174-189.

B. Huppert (1967), Endliche Gruppen I (Springer-Verlag, Berlin 1967).

S. Lang (1956), 'Algebraic groups over finite fields', Amer. Math. J. 78, 555-563.

E. T. Parker, 'A simple group having no multiply transitive representation', Proc. Amer. Math. Soc. 5, 606-611.

Bhama Srinavasan (1968), 'The characters of the finite symplectic group Sp (4, q)', Trans. Amer. Math. Soc. 131, 488-425.

J. Tits (1964), 'Algebraic and abstract simple groups', Ann. of Math. 80, 313-339.

E. Witt (1937), 'Die 5-fach transitiven Gruppen von Mathieu', Abh. Math. Sem. Univ. Hamburg 12, 256-264.

Department of Pure Mathematics

University of Adelaide

South Australia 